\title{
A Novel Approach for Plant Identification
}

\author{
Sonali A. Kanade \\ Student of BE Computer \\ Engineering \\ BVCOE \& RI, Nashik, India, \\ University of Pune
}

\author{
Ashok R. Ambore \\ Student of BE Computer \\ Engineering, BVCOE \& RI \\ Nashik, India, University of Pune
}

\author{
Chaitali S. Patil \\ Student of BE Computer \\ Engineering \\ BVCOE \& RI, Nashik, India, \\ University of Pune
}

\author{
Tejaswini S. Pawar \\ Student of BE Computer \\ Engineering \\ BVCOE \& RI, Nashik, India, \\ University of Pune
}

\author{
Hemant D. Sonawavne \\ Head of Computer Engineering \\ BVCOE \& RI,Nashik \\ India, University of Pune
}

\begin{abstract}
Plant Identification has a place with a particular application space of data mining. Pictures of plant leaves are generally utilized as the primary component to recognize a plant from another. For legitimate recognizable proof, highlight extraction is essential. In the literature, most plant acknowledgment frameworks utilize the highlights alongside a grouping technique, which has been adjusted or changed to confront this kind of application. In this paper, we are building up a portable application for distinguishing restorative plants which utilizes Profound Learning and Convolutional Neural System to take in discriminative highlights from leaf pictures with classifiers for plant ID. We propose three new geometric highlights that depict the vertical and level symmetry of clears out. These highlights are easy to separate from pictures. As per the consequences of trials, when these highlights are utilized as a part of conjunction with other surely understood geometric attributes, the execution of traditional order strategies is amazingly moved forward. To demonstrate the adequacy of the proposition, We test few classifiers with pictures of leaves openly accessible on We, This framework will manage professionals of Ayurveda and ordinary android clients too.
\end{abstract}

\section{Keywords}

Identification, Dataset, Feature Extraction, Plant Recognition, geometric feature

\section{INTRODUCTION}

In this paper, we are building up a portable application for distinguishing restorative plants which utilizes Profound Learning and Convolutional Neural System to take in discriminative highlights from leaf pictures with classifiers for plant ID. Given a picture of leaves of any types of Restorative Plants, the framework will have the capacity to distinguish and recognize the types of regarded plant and its area. Application will require a photo of leaf of plant of any size, determination, taken whenever of day for handling the outcome. Framework will contrast the information picture and preparing dataset. Our framework acquires best in class execution on this present reality pictures from the new Dataset made by catching pictures of clears out. This framework will manage professionals of Ayurveda and ordinary android clients too.

Plant ID is imperative in zones, for example, medication, organic science, nursery and nourishment area. It is additionally important and interesting for environmentalists, home grown specialists and overall population. In any case, exact plant acknowledgment is an errand that requires some plant mastery. The development of plant recognition methods is an active research area in last year's. Most of plant identification methods use leaf due to its attractive properties, for example, leaf shape varies between different species, and leaves can be collected throughout the year. Typically, some features are extracted from images of leaves, and then data mining methods are applied to recognize plants.

\section{METHODOLOGY}

In this section, the general procedure for plant distinguishing proof comprises of the following advances:

1. Pre-processing-this field includes scaling, normalization and segmentation of images.

2. In Feature extraction which consists in acquiring geometric, shape or some other sort of highlights from images of leaves.

3. Classification Application of data mining methods which sometimes adapted to this particular application to identify new leaves. In the following subsections, we explain the general process to apply current classification methods for plant identification.

A Pre-processing and feature extraction- The procedure for separating the highlights from leaves requires that all pictures have a similar size. Be that as it may, the vast majority of leaf databases accessible on the Web contain pictures with various measurements. In this way, we scale the pictures to a size of $1000 \times 600$ pixels. Keeping in mind the end goal to remove valuable highlights from a shading picture of a leaf, it is important to change it into a top notch double picture. Normalization and segmentation are usually used for this purpose.

Image normalization: is utilized to have a specific freedom of the properties of the picture, for example, brilliance and differentiate. This requires to ascertain the bigger and littler pixel qualities, max (I) and min (I), separately. We utilize condition (1) to standardize the picture:

$$
N(x, y)=\frac{255(\mathrm{I}(\mathrm{X}, \mathrm{Y})-\min (\mathrm{I}))}{\max (\mathrm{I})-\min (\mathrm{I})}
$$

Leaf segmentation: 
We use a spatial thresholding method to obtain a binary image of leaves. Thethreshold $\mathrm{T}(\mathrm{x} ; \mathrm{y})$ is calculated at every pixel, it relies upon the probability distribution and/or correlation between pixels. The equation (2) is used for this purpose.

$\mathrm{T}(\mathrm{x}, \mathrm{y})\{1 \mathrm{~T}(\mathrm{x}, \mathrm{y})>\mathrm{U}$

0 otherwise $\}$

Where $U=\bar{x}$ is the arithmetic mean of the whole image. Once the threshold is computed, it is necessary to eliminate the noise present in the image, and also close the holes that exist within the image. This is done using the morphological opening operation, with a mask of $3 \times 3$ pixels.

Contour extraction: Extraction of geometric features of leaves usually require a clear contour of leaf. The parallel picture got in past advance gives a district of pixels of the leaf, which can be utilized to concentrate of helpful highlights for order. We propose a basic technique to acquire the form. It is as per the following: for every pixel of the picture, break down an area of $3 \times 3$ pixels, and compute the pixel of least event, it is viewed as the limit $U$. Then, it is used again into equation (2), thereby obtaining only the outline of the binary image.

Feature extraction:Highlight extraction is a standout amongst the most essential stages in plant acknowledgment. There exist a few surely understood highlights for plant acknowledgment. The most Well-known are the accompanying

$$
\begin{aligned}
& \text { Convexity Leaf }=\frac{\text { Area Of Leaf }}{\text { Area Of Hull Leaf }} \\
& \text { Area Ratio }=\frac{\text { Length Of Largest Axis }}{\text { Length Of Smallest Axis }}
\end{aligned}
$$

\section{RELATED WORK}

The extraction of highlights from leaf is one of the essential ventures in plant distinguishing proof. Along these lines, numerous analysts have proposed highlights that aim to catch the principle qualities of takes off. An organized portrayal of groupings for leaf form is appeared in [1]. The portrayal depends on ebb and flow find in the leaf edge. It de-relates the data display on a leaf edge from alternate states of it.In [5], authors propose following features which are invariant to scale, these are the accompanying: perspective proportion, spanaspect ratio, radius ratio and slice ratio. Here, Authors tested the usefulness of their features with only one classification method, support vector machine. In this paper,Five main morphological features Were proposed in [6].These describe biological characteristics of leaf, (the perimeter (PRI), the area (PXC), the major ellipse axis (MJX), the minor ellipse axis (MNX) and the logarithm of the height to width ratio (LHW). These features are extracted from double pictures acquired in a division step. A comparative approach is displayed in [7]. They register the accompanying highlights of leaf: length and width, range, border, body region, structure border, removes outline the vertical and level, shading histogram and centroid-based outspread separation distance map. Three extra morphological highlights got from two half areas of leaf are introduced in [8]. In that work, a leaf is divided and every half is utilized to register region proportion, degree proportion and rectangularity proportion.

\section{SYSTEM ARCHITECTURE}

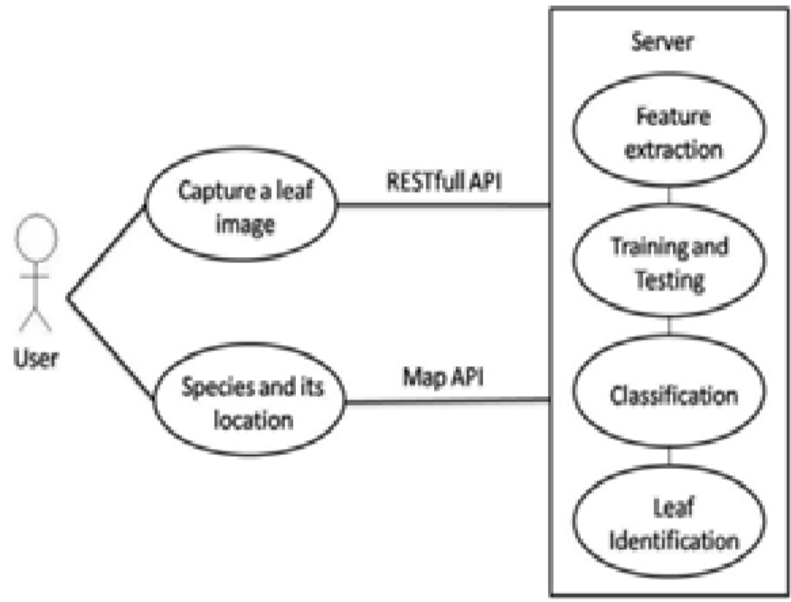

Fig 1: System Architecture

In this Architecture diagram, we are developing portable application for plant Identification. Figure 1 shows the flow of whole system which contain user, camera for capturing image and server for storing data.

\section{Serialisation of steps:}

A.Firstly, User will capture a leaf image of plant and pass to the server, as Well as species its location.

B.Feature Extraction field extract the all features of leaf and it will pass to the training.

C..In training and testing field, all features of leaf are trained by using support vector machine.

D.In classification, trained and tested data will be grouping for maintain their accuracy using naivebays algorithm,

E.After classified, it will pass to leaf identification for recognizing accuracy of leaf.

\section{ALGORITHM}

\subsection{Naive Bayes Algorithm}

It is a classification technique based on Bayes' Theorem with an assumption of independence among predictors. In simple terms, a Naive Bayes classifier assumes that the presence of a particular feature in a class is unrelated to the presence of any other feature.

For example, a leaf may be considered to be a Tulsi if it is green, curve, and about 1 inch in diameter. Even if these features depend on each other or upon the existence of the other features, all of these properties independently contribute to the probability that this leaf is a Tulsi and that is why it is known as 'Naive'.

Naive Bayes model is easy to build and particularly useful for very large data sets. Along with simplicity, Naive Bayes is known to outperform even highly sophisticated classification methods.

Bayes theorem provides a way of calculating posterior probability $\mathrm{P}(\mathrm{c} \mid \mathrm{x})$ from $\mathrm{P}(\mathrm{c}), \mathrm{P}(\mathrm{x})$ and $\mathrm{P}(\mathrm{x} \mid \mathrm{c})$. Look at

The Equation is below- 


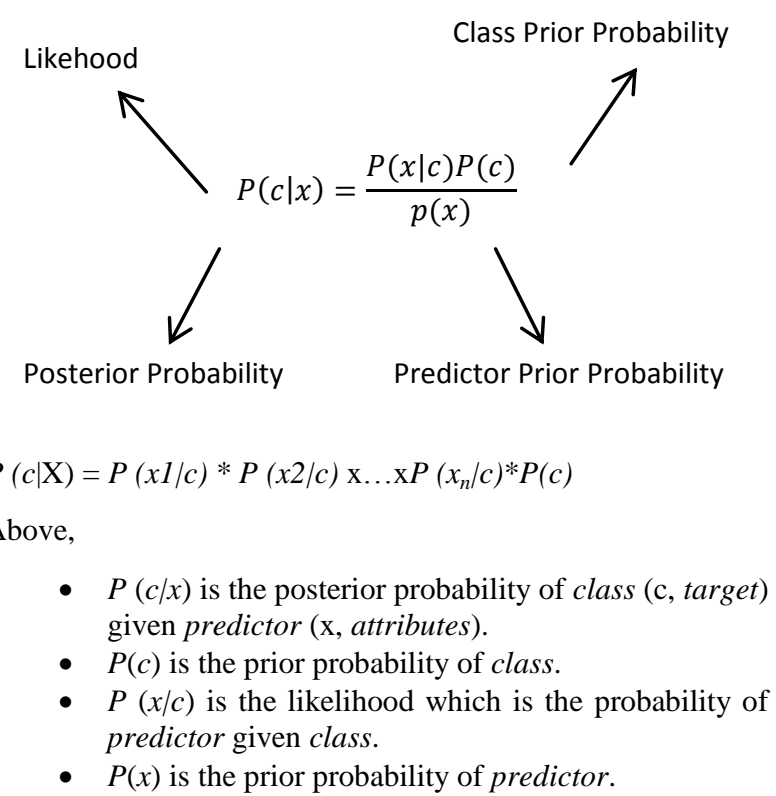

\section{CONCLUSION}

Hence in We, a new approach of using deep learning method was explored in order to automatically classify from leaf pictures. The created model could identify leaf presence and distinguish between healthy leaves, which can be visually analyzed. The entire procedure was described, respectively, from gathering the pictures used for training and validation to image preprocessing and augmentation and finally the procedure of training the deep $\mathrm{CNN}$ and and fine tuning. Distinctive checks were performed in order to check the performance of newly created model. An extension of this study will be on gathering images for enriching the database and improving accuracy of the model using different techniques of fine-tuning and augmentation. We propose three new geometric highlights that depict the vertical and level symmetry of clears out. These highlights are easy to separate from pictures. As per the consequences of trials, when these highlights are utilized as a part of conjunction with other surely understood geometric attributes, the execution of traditional order strategies is amazingly moved forward. To demonstrate the adequacy of the proposition, We test few classifiers with pictures of leaves openly accessible onWe. This framework will manage professionals of Ayurveda and ordinary android clients too.

\section{REFERENCES}

[1] G. Cerutti, L. Tougne, D. Coquin, and A. Vacavant, "Leaf margins as sequences: A structural approach to leaf identification," Pattern Recognition Letters, vol. 49, pp. $177-184,2014 . \quad$ [Online]. Available: http://www.sciencedirect.com/science/article/pii/S01678 65514002335

[2] J. S. Cope, D. Corney, J. Y. Clark, P. Remagnino, and P. Wilkin, "Plant species identification using digital morphometrics: A review," Expert Systems with Applications, vol. 39, no. 8, pp. 7562 - 7573, 2012. [Online].

Available: http://www.sciencedirect.com/science/article/pii/S09574 17412000863

[3] C.-Y. Gwo, C.-H.Wei, and Y. Li, "Rotary matching of edge features for leaf recognition," Computers and Electronics in Agriculture, vol. 91, pp. 124 - 134, 2013.[Online].
http://www.sciencedirect.com/science/article/pii/S01681 69912002906

[4] C. Zhao, S. S. Chan, W.-K. Cham, and L. Chu, "Plant identification using leaf shapes-a pattern counting approach," Pattern Recognition, vol. 48, no. 10, pp. 3203 - 3215, 2015, discriminative Feature Learning from Big Data for Visual Recognition. [Online]. Available: http://www.sciencedirect.com/science/article/pii/S00313 20315001284

[5] S. Watcharabutsarakham, W. Sinthupinyo, and K. Kiratiratanapruk, "Leaf classification using structure features and support vector machines,"in Information Science and Service Science and Data Mining(ISSDM), 2012 6th International Conference on New Trends in. IEEE, 2012.

[6] J. I. Arribas, G. V. S'anchez-Ferrero, G. Ruiz-Ruiz, and J. G'omez-Gil, "Leaf classification in sunflower crops by computer vision and neural networks," Computers and Electronics in Agriculture,vol. 78, no. 1, pp.9-18,2011. [Online].

Available: http://www.sciencedirect.com/science/article/pii/S01681 69911001220

[7] T. Munisami, M. Ramsurn, S. Kishnah, and S. Pudaruth, "Plant leaf recognition using shape features and colour histogram with k-nearest neighbor classifiers," Procedia Computer Science, vol. 58, pp. $740-747,2015$, second International Symposium on Computer Vision and the Internet (VisionNet15). [Online]. Available: http://www.sciencedirect.com/science/article/pii/S18770 50915022061.

[8] Uluturk and A. Ugur, "Recognition of leaves based on morphological features derived from two half-regions," in Innovations in Intelligent Systems and Applications (INISTA), 2012 International Symposium on, July 2012, pp. $1-4$

\section{AUTHOR'S PROFILE}

Sonali A.Kanade she is Engineering student of Computer Engineering at Brahma Valley College of Engineering And Research Institute, Nasik under University of Pune. Her interest in the field of Innovation in Application.

Chaitali S.Patil she is Engineering student of Computer Engineering at Brahma Valley College of Engineering and Research Institute, Nasik under University of Pune. Her interest in the field of Innovation in Application.

Tejaswini S.Pawar she is Engineering student of Computer Engineering at Brahma Valley College of Engineering and Research Institute, Nasik under University of Pune. Her interest in the field of Innovation in Application.

Ashok R.Ambore he is Engineering student of Computer Engineering at Brahma Valley College of Engineering And Research Institute, Nasik under University of Pune. His interest in the field ofInnovation in Application.

Hemant D.Sonawavne, ME, BE Computer Engg. Was educated in Pune University. Presently he is working as a Head of Computer Department of Brahma Valley College of Engineering and Research Institute, Nasik, Maharashtra, India.he has presented papers at National and International conferences and also published papers in National and International Journals on various aspects of Computer Engineering and Networks. His areas of interest include Computer. 